\title{
Study on the change characteristics of regional geoelectric fields
}

\author{
Y Y FAN ${ }^{1,2}, \mathrm{Z} \mathrm{H} \mathrm{AN}{ }^{1,2, *}, \mathrm{~J} \mathrm{~J} \mathrm{WANG}^{1,2}$ and $\mathrm{J} \mathrm{LiU}^{1,2}$ \\ ${ }^{1}$ Lanzhou Institute of Seismology, China Earthquake Administration, Lanzhou, The People's Republic of China. \\ ${ }^{2}$ Earthquake Administration of Gansu Province, Lanzhou, The People's Republic of China. \\ *Corresponding author. e-mail: anzhanghui5@hotmail.com
}

MS received 20 March 2018; revised 12 October 2018; accepted 24 November 2018; published online 22 March 2019

In this study, we illustrate the temporal variations of the geoelectric field and geomagnetic field by data from eight geoelectric field stations and four geomagnetic field stations in Mainland China in 2011. The maximum entropy spectral estimation method (MEM) was used to process the data in our study. The results show that: (i) it is obvious seasonal changes at inland geoelectric field stations and monthly changes at coastal geoelectric field stations; (ii) obvious seasonal changes can be observed at geomagnetic field stations and its seasonal changing curve of component $\mathrm{H}$ is most similar to that of the geoelectric field; and (iii) monthly changes of geoelectric field are caused by the combined action of solar and lunar tidal forces, and the seasonal changes of geoelectric field are caused by electromagnetic induction of the seasonal changes of ionospheric current induction intensity inside the Earth.

Keywords. The change characteristics; geoelectric field; geomagnetic field; the Earth; the Sun; the Moon.

\section{Introduction}

At the beginning of the 20th century, the national and international scholars had observed and studied the geoelectric field of fixed stations aiming at monitoring and forecasting the disaster events such as earthquake and studying the electromagnetic environment of the Earth, but they obtained limited results that are limited by the observation technologies and accumulated data (KPAER 1951; Varotsos and Alelopoulous 1984). In recent years, the national and international scholars make use of geoelectric field data observed by fixed stations to mainly devote to monitoring and forecasting disaster events such as earthquake (Uyeda 1995; Mogi et al. 2000; Nagao et al. 2000; Uyeda et al. 2000, 2002; Zlotnicki et al. 2001; Ma et al. 2004;
Enomo et al. 2006; Nagamoto et al. 2008; Fan et al. 2010; Liu et al. 2011; Orihara et al. 2012; Huang et al. 2017), and magnetotelluric measurements have been used to research the electrical conductivity of the crust and upper mantle (Niwas et al. 2005; Tang et al. 2005; Israil et al. 2008; Xiao et al. 2009; Zhan et al. 2013; Miglani et al. 2014). Mogi et al. (2000) researched the relationship between electric field changes and earthquake, and short-term electric field variations were found to correspond mainly to the geomagnetic activity, while the long-term variation was mostly a gradual shift and not clearly correlated with neither precipitation nor groundwater level variation. Nagao et al. (2000) proved that co-seismic signals do exist, but they started with the arrival of seismic waves and not at the origin time of earthquakes. Ma et al. 
(2004) investigated the change of 18 geoelectric field observation stations around the south-north seismic belt in China and found that abnormal electromagnetic disturbance signals are recorded at five stations before the 20 April 2013 Lushan Ms 7.0 earthquake. Overall, the scholars from Japan and Indonesia analysed the seismic signal used for the raw data observed by different device system, but the Chinese scholar used different signal processing methods to analyse the seismic signal observed by the same device system.

Huang et al. (2017) studied the earthquake precursors in China for recent 50 yrs, and the ground-based electromagnetic observation related to earthquakes in China has been established after the occurrence of the large Tangshan Ms 7.6 earthquake, on 28 July, 1976. Basically, it is divided into geomagnetic field observation, geoelectric field observation and electromagnetic perturbation observation, highlighting the 4 February 1975 Haicheng earthquake which was successfully predicted. The data observed by the geoelectric field observation is determined by observation methods, technologies, electromagnetic environment and site conditions (Qian et al. 2004; Du et al. 2006). Geoelectric field curves for the earthquakes in Mainland China are diverse and complex. Through collecting the observation data of 122 stations in Mainland China, it is found that about one-third of the stations present relatively stable peak-valley daily changes, and scholars have done a plenty of research on daily changes of the geoelectric field and its mechanism of production (Huang and Liu 2006; Zhang et al. 2007; Tan et al. 2010), and they have received welcome results, but the research on monthly and seasonal changes of the geoelectric field is almost in blank.

It is an active demand for the theory, method and application of the geoelectric field to recognise the change characteristics and mechanism of the geoelectric field. In this paper, with changes of the observation data in 2011 of four geoelectric field stations near Qinghai-Tibet Plateau and four geoelectric field stations along the eastern coastal regions in Mainland China as the object of study compares with the observation data of geomagnetic field, monthly and seasonal changes of geoelectric field as well as the interrelation with the Moon revolving around the Earth and the Earth revolving around the Sun are studied, and expect to have an initial understanding of the cause of monthly and seasonal changes of geoelectric field, so as to help to recognise complex short-impending electromagnetic phenomena of earthquake. The geoelectric/geomagnetic field is affected by electromagnetic field in the ionosphere because of electromagnetic induction, and geomagnetic activity affects almost all ionospheric parameters (Danilov and Lastoviks 2001). The short-term electric field variations correspond mainly to geomagnetic activity (Mogi et al. 2000) and the data has been deleted when the geomagnetic activity is strong during the computing of monthly and seasonal changes of geoelectric/geomagnetic field (Zeng et al. 2009; Liu et al. 2011).

\section{Basic condition of stations and data processing method}

\subsection{Basic condition of stations}

China is the only country that makes a large-scale normalised geoelectric observation (Du et al. 2006). There are 122 geoelectric field stations built by the China Earthquake Administration spreading all over Mainland China, and there are more in the east, fewer in the west, more near fault zones but fewer near non-fault zones.

The two densest regions for geoelectric field observation in Mainland China include: (i) Qinghai-Tibet Plateau and its surrounding area: In recent years, moderate-to-strong earthquakes often take place here, such as Yutian Ms 7.3 earthquake $\left(\mathrm{N} 35.6^{\circ} ; \mathrm{E} 81.6^{\circ}\right)$ on 21 March 2008, Wenchuan Ms 8.0 earthquake $\left(\mathrm{N} 31.0^{\circ} ; \mathrm{E} 103.4^{\circ}\right)$ on 12 May 2008, Yushu Ms 7.1 earthquake (N33.1; E96. $7^{\circ}$ ) on 14 April 2010 and Lushan Ms 7.0 earthquake $\left(\mathrm{N} 30.3^{\circ}\right.$ and E103. $0^{\circ}$ ) on 20 April 2013. The China Earthquake Administration has built nearly 40 geoelectric field stations at Qinghai-Tibet Plateau and its surrounding area to monitor the seismic activities there, and it is the densest among inland areas. (ii) Eastern of Mainland China: It has nearly 50 geoelectric field stations built by the China Earthquake Administration, and it is the densest in Mainland China. Pingliang $\left(\mathrm{N} 35.6^{\circ} ; \mathrm{E} 106.7^{\circ}\right)$, Gufeng $\left(\mathrm{N} 37.4^{\circ} ; \mathrm{E} 102.8^{\circ}\right)$, Shandan $\left(\mathrm{N} 38.8^{\circ} ; \mathrm{E} 101.1^{\circ}\right)$ and Guazhou $\left(\mathrm{N} 40.5^{\circ} ; \mathrm{E} 95.8^{\circ}\right)$ geoelectric field stations are located in the north and northeast of Qinghai-Tibet Plateau; Changli (N39.7 ${ }^{\circ}$ E119.1 $\left.{ }^{\circ}\right)$, Malingshan $\left(\mathrm{N} 34.7^{\circ} ; \mathrm{E} 118.5^{\circ}\right)$, Gaoyou (N32.7 ; E119. $\left.3^{\circ}\right)$ and Haian $\left(\mathrm{N} 32.5^{\circ} ; \mathrm{E} 120.5^{\circ}\right)$ geoelectric field stations are located at eastern coastal China, and the geoelectric field data observed by those 


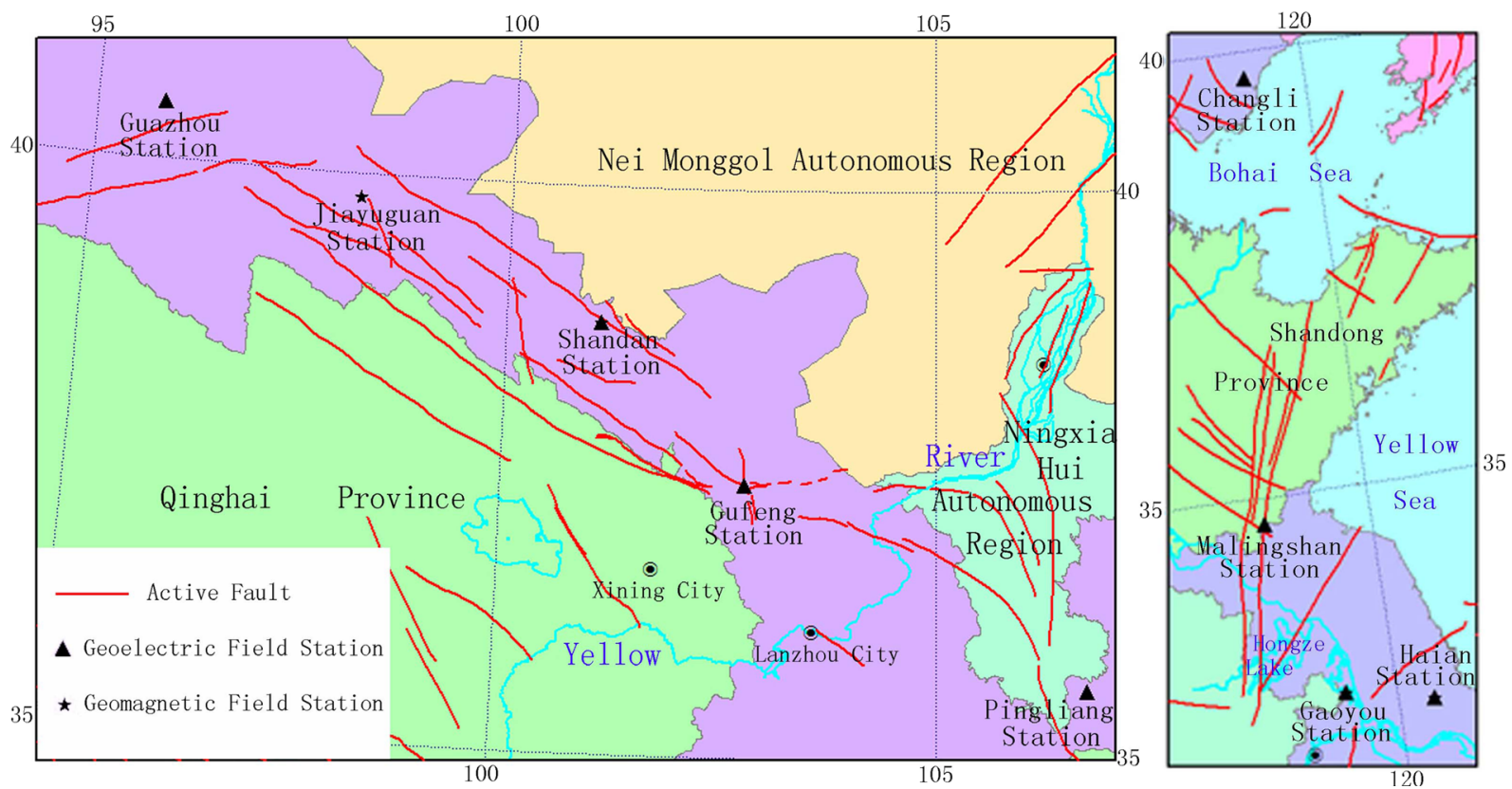

Figure 1. Distribution of stations.

stations are collected to study the monthly and seasonal change characteristics, and then compare with the geomagnetic field data of inland stations included Jiayuguan $\left(\mathrm{N} 39.8^{\circ} ; \mathrm{E} 98.2^{\circ}\right)$ and Shandan $\left(\mathrm{N} 38.8^{\circ} ; \mathrm{E} 101.1^{\circ}\right)$ as well as coastal stations included Gaoyou $\left(\mathrm{N} 32.7^{\circ} ; \mathrm{E} 119.3^{\circ}\right)$ and Haian $\left(\mathrm{N} 32.5^{\circ} ; \mathrm{E} 120.5^{\circ}\right)$ to analyse monthly and seasonal change characteristics of regional geoelectric fields, and expect to get the influences of underground structure and observation environments on the change characteristics of geoelectric field, and also hope to get the cause of geoelectric field abnormality at earthquake. The distribution of stations is shown in figure 1.

Pingliang, Gufeng, Shandan and Guazhou geoelectric field stations located at the west of Mainland China, northeast and north of Qinghai-Tibet Plateau, all of which have an elevation of over $1000 \mathrm{~m}$ but poor underground water system, especially for Shandan Station having Gobi Desert on the surface but almost no vegetation coverage. The above four stations have been used to study Wenchuna, Yutian, Yushu and some other earthquakes as well as to study cyclic changes of geoelectric field for many times (Fan et al. 2010, 2014; Tan et al. 2010, 2012; An et al. 2013). Changli, Malingshan, Gaoyou and Haian geoelectric field stations are located at eastern coastal Mainland China and Changli Station has an elevation of $18 \mathrm{~m}, 20 \mathrm{~km}$ away from the coastline; Malingshan Station has an elevation of $110 \mathrm{~m}, 70 \mathrm{~km}$ away from the coastline; Haian
Station has an elevation of $4 \mathrm{~m}, 40 \mathrm{~km}$ away from the coastline; and Gaoyou Station has an elevation of $4 \mathrm{~m}, 150 \mathrm{~km}$ away from the coastline. Gaoyou Station has the richest water system among the four stations, because the observation area of Gaoyou Station has some lakes nearby, such as Gaoyou Lake and Hongze Lake. The above stations conform to the construction norms of geoelectric field stations (Qian et al. 2004; Du et al. 2006), setting three observation directions along the NS (north-south), EW (east-west) and slant, with good electromagnetic environment around the stations and high data observation quality, and without obvious interference source. The sampling period of geoelectric field data is $1 \mathrm{~min}$. Setting components H, D and F observation, Shandan, Gaoyou, Haian and Jiayuguan geomagnetic field stations have a sampling period of $1 \mathrm{~min}$. Among them, the geoelectric and geomagnetic field stations of Shandan, Gaoyou and Haian share the same observation area, whose surveying area and surrounding will not be repeated; Jiayuguan geomagnetic field station located at the northeastern Tibetan Plateau, having an elevation of $1700 \mathrm{~m}$ with dry and flat observation area without obvious interference source.

In 2011, no major earthquakes happened at Qinghai-Tibet Plateau and its surrounding area, and its seismic activity is relatively stable, and a relatively peaceful background basis is provided for the study of monthly and seasonal changes of the geoelectric field. 


\subsection{Data processing method}

In this paper, maximum entropy spectral estimation method (MEM) is used as a computing method. The basic idea of MEM refers to: supposing all the data except the limited data observed to be random, under the premise of information entropy is the maximum, the part of unknown correlation functions is deduced with an iterative method to get a power spectrum. In this way, the error of spectrum estimation will be reduced and the resolution ratio will be greatly increased, because the sequence length of correlation functions is increased.

For a stable random sequence $\{X n\}$, the problem to compute the maximum entropy spectrum is to find the relation between the auto-correlation function and power spectrum density (PSD) that meets the constraint condition to obtain limited auto-correlation functions with given data and to obtain maximum entropy PSD with variation principle:

$$
\hat{S}_{x}(f)=\frac{P_{M}}{2 f_{c}\left|1+\sum_{m=1}^{M} a_{m} \mathrm{e}^{-j 2 \pi m f T}\right|^{2}} .
$$

In the above equation, $f$ is frequency, $a_{m}$ is prediction error coefficient, $P_{M}$ is prediction error power and $M$ is filter order. The selection of $M$ plays a crucial part in maximum entropy spectrum estimation. The resolution of spectrum estimation can be improved, line splitting can be eliminated, etc, through selecting the right order of model (Song et al. 1997). In this paper, the FPE (final prediction error) criterion is used to determine $M$ (Wang 1997):

$$
F_{(\mathrm{Pe})_{M}}=\frac{N+M+1}{N-M-1} P_{M}
$$

$P_{M}$ decreases with the increasing of $M, N$ is the number of values passed in, but $(N+M+1) /$ $(N-M-1)$ increases with the increasing of $M$. $F_{(\mathrm{Pe})_{M}}$ will reach the minimum at $M=M_{\mathrm{opt}}$, and this $M_{\text {opt }}$ will be regarded as the optimal order $M$ concluded from $F_{(\mathrm{Pe})}$ criterion. Under normal circumstances, limiting $M_{\max } \leq N / 2$, the following empirical equation should be used to define $M$ :

$$
M= \begin{cases}{\left[\frac{N}{3}-1\right] \sim\left[\frac{N}{2}-1\right],} & 20 \leq N \leq 100 \\ (0.05 \sim 0.2) N, & N>100\end{cases}
$$

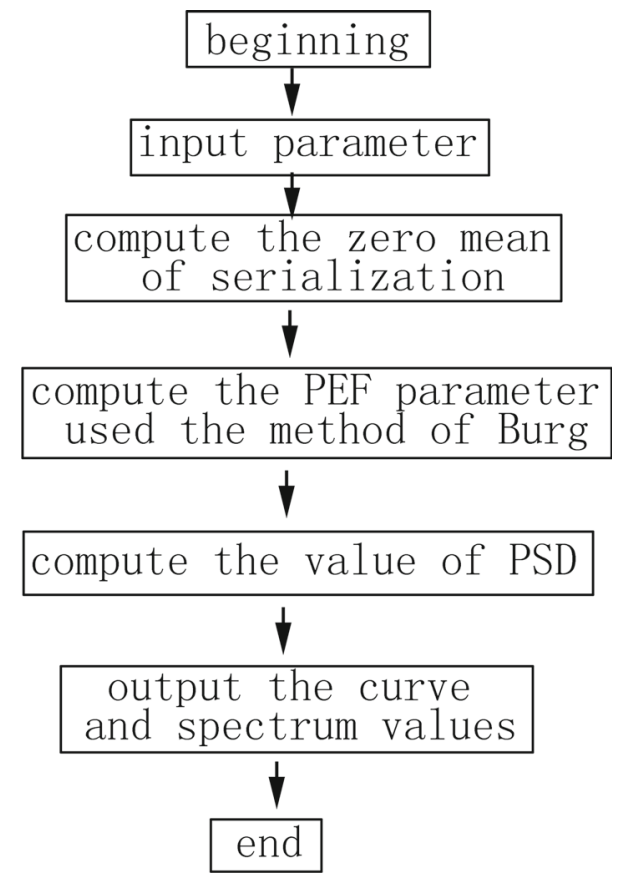

Figure 2. The calculation flow diagram of maximum entropy estimation.

The calculation flow diagram of maximum entropy estimation is shown in figure 2.

This method has been used in analysing the distortion and abnormality of the geoelectric field before and after Wenchuan, Yutian and Yushu earthquakes for several times and in studying the geoelectric/geomagnetic field of Sino-French international cooperation station near several moderate earthquakes in Gansu Tianzhu area (Fan et al. 2010, 2015; Liu et al. 2011). The results indicate that the obvious increase and decrease will occur due to low-frequency component PSD value of geoelectric/geomagnetic field $20 \sim X$ days before the earthquake, and MEM can well reflect the frequency spectrum information of geoelectric/geomagnetic field.

\section{Magnetic analysis}

Special attention should be paid to the magnetic influence when analysing the electric field changes. In this study, the indexes $K_{\mathrm{p}}$ and $D_{\mathrm{st}}$ were used. The $K_{\mathrm{p}}$ index was used to describe the average geomagnetic activity intensity over $3 \mathrm{~h}$, and it ranged from 0 to 9 . The sum of eight $K_{\mathrm{p}}$ values is denoted as $\sum K_{\mathrm{p}}$, and values of $\sum K_{\mathrm{p}}>30$ are associated with intense geomagnetic activities of that day (Hattori et al. 2002). The $D_{\text {st }}$ index monitors the intensity of the global ring current induction 

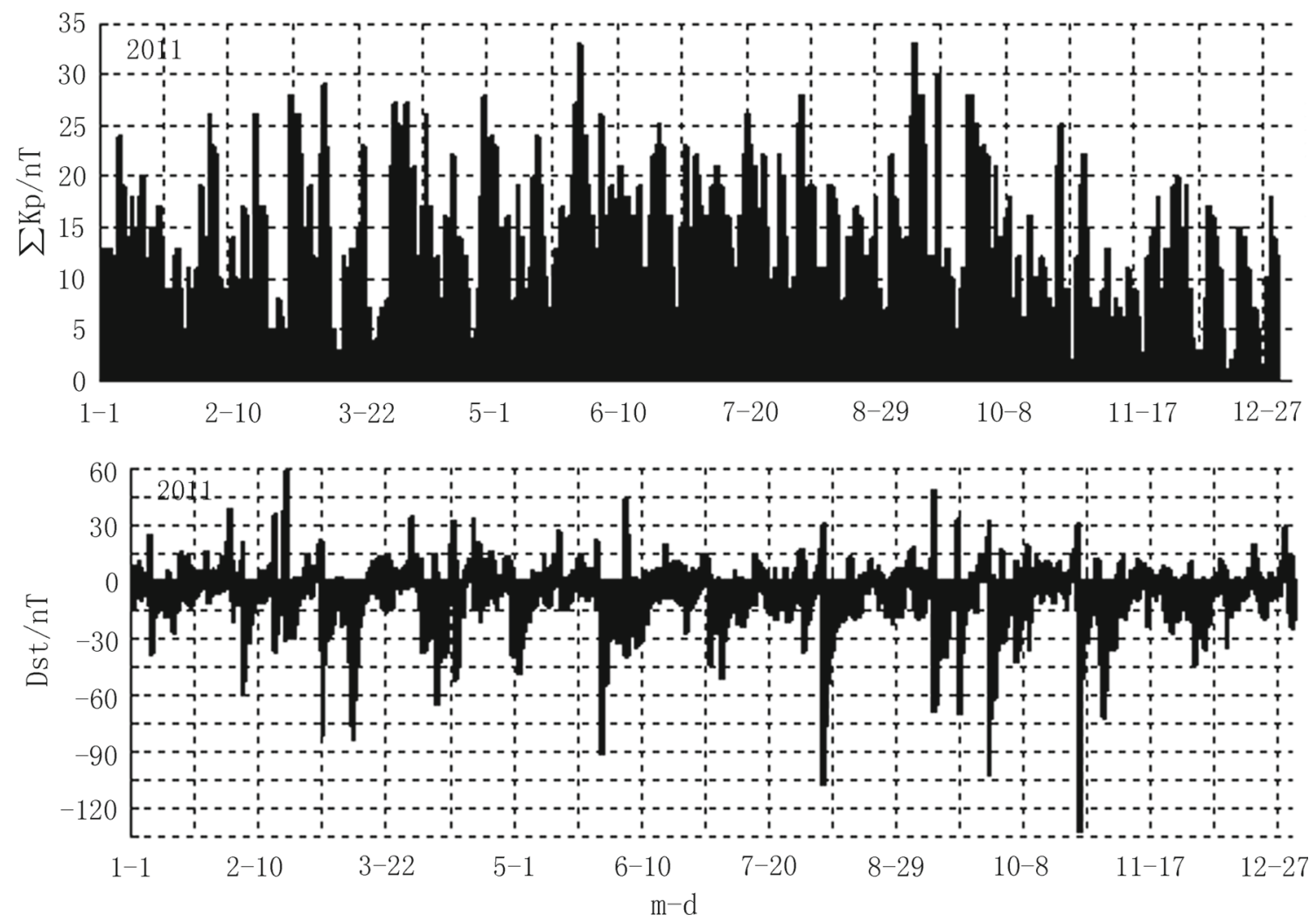

Figure 3. Geomagnetic indexes $K_{\mathrm{p}}$ and $D_{\text {st }}$ in 2011.

field, and in general, a magnetic storm will likely take place when the $D_{\text {st }}$ value is smaller than $-50 \mathrm{nT}$ and such a condition lasts for two or more hours (Gonzalez et al. 1994). In this study, the study period of the geoelectric/geomagnetic observation data included the entire year of 2011, and the time spans of indexes $K_{\mathrm{p}}$ and $D_{\text {st }}$ were the same as that of the geoelectric field observation data (see figure 3 for details). While using the observation data of geoelectric field and geomagnetic field, the data is deleted when $\sum K_{\mathrm{p}}>30$ or the $D_{\text {st }}$ value is smaller than $-50 \mathrm{nT}$ and such a condition lasts for two or more hours (Zeng et al. 2009; Liu et al. 2011). The $K_{\mathrm{p}}$ values were obtained from http://www-app3.gfz-potsdam.de/ obs/niemegk/monrep/index.html and the $D_{\text {st }}$ values were obtained from http://wde.kugi.kyoto-u. ac.jp/dst_realtime.

\section{Monthly and seasonal changes of the geoelectric/geomagnetic field}

\subsection{Geoelectric field stations}

Figure 4 shows the PSD value changes with the time for inland Pingliang, Gufeng, Shandan and
Guazhou geoelectric field stations in 2011 computed with MEM, and $X$-coordinate represents the date and $Y$-coordinate represents the average value of PSD (The range of frequencies considered in this density spectrum is from 0 to $0.00832175 \mathrm{~Hz}$, the $Y$-coordinate is the average of PSD whose frequency is from 0 to $0.00832175 \mathrm{~Hz}$ ). As shown in the figure, the average value of PSD measured along the NS, EW and NE at Pingliang Station increased synchronously from the beginning of March 2011, until the early November, and such an increase disappeared, lasting for approximately 250 days, taking $68.49 \%$ of the whole year. The average value of PSD reached maximum values in April and September, and it seems to fluctuate from March to May and from August to early November. However, there was no apparent PSD fluctuation in June and July (see figure 4A for details).

Figure 4(B and C) shows the MEM maps of the geoelectric field from January to December 2011 at Gufeng Station and Shandan Station, respectively, sharing the same calculation methods and same mapping fields as in figure 4(A). Both Gufeng Station and Shandan Station had the phenomena of Pingliang Station, as the average value of PSD was 
large in summer and small in winter with increasing and decreasing intervals lasting the same time and the fluctuation curves almost in coincidence.
Figure 4(D) shows the MEM map of Guazhou geoelectric field station that is relatively far away from the above three stations in geographical
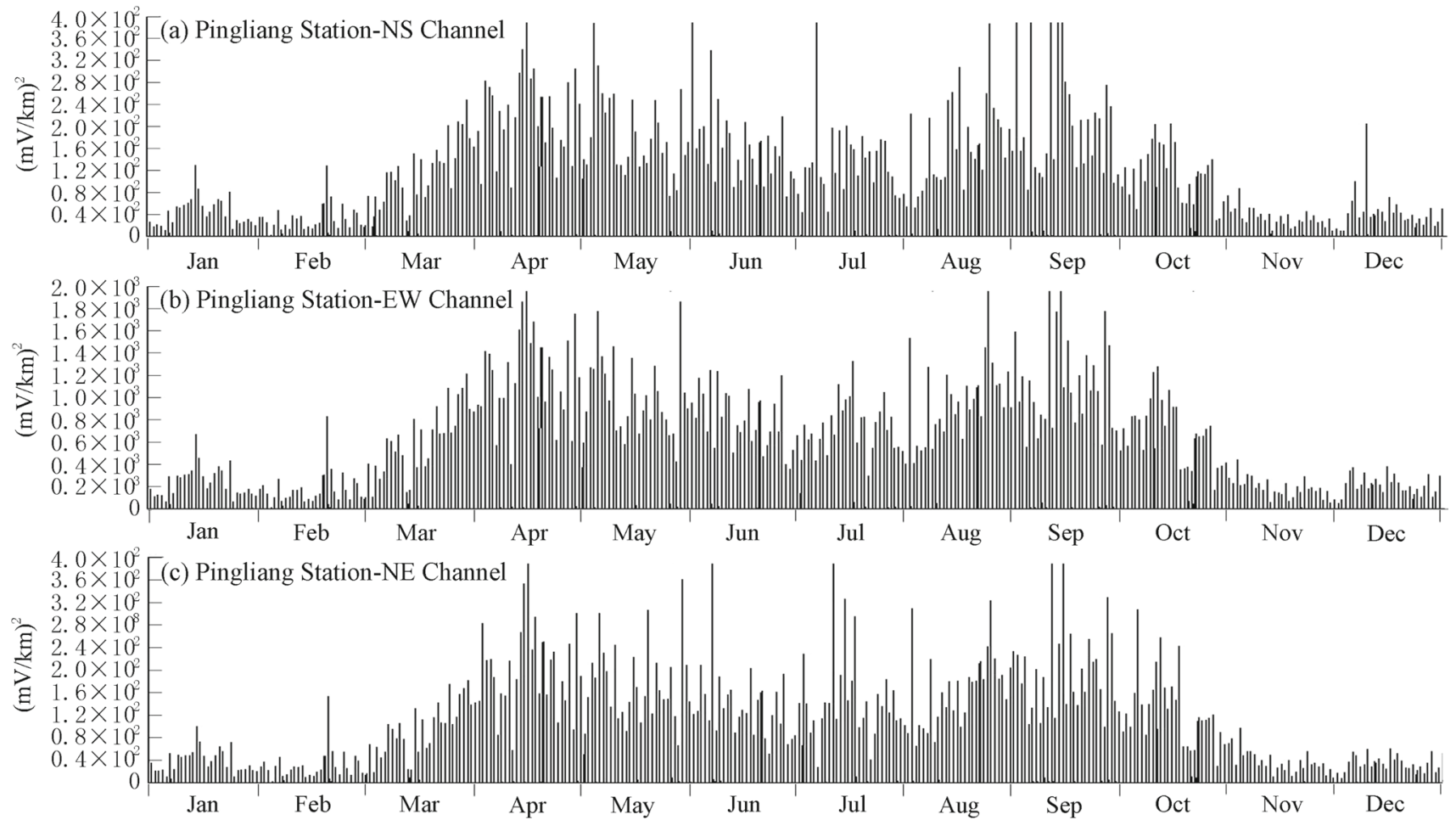

(A)
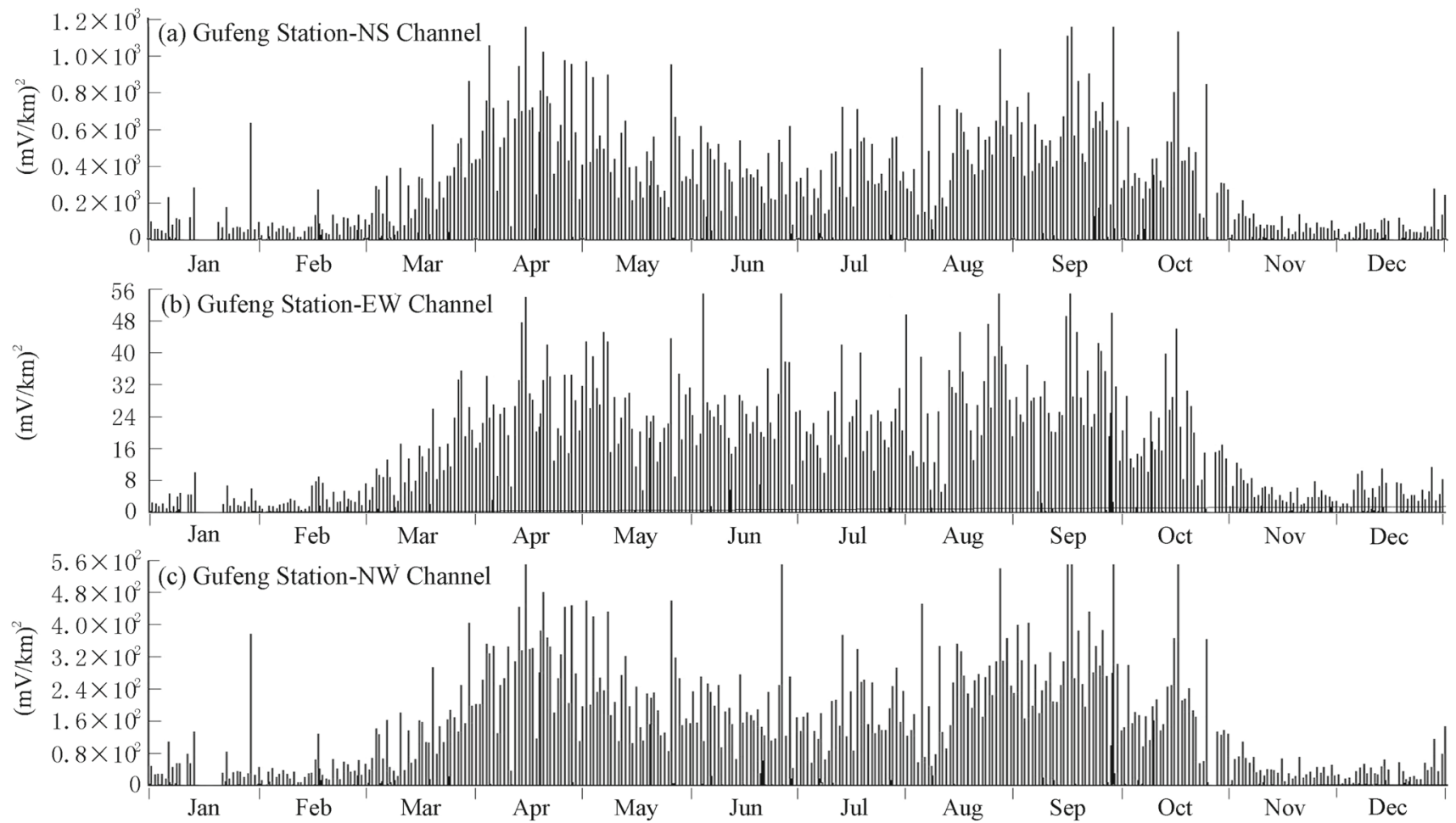

(B)

Figure 4. The map of the averaged PSD for the frequency range 0-0.00832175 Hz of geoelectric fields at the Pingliang (A), Gufeng (B), Shandan (C) and Guazhou (D) stations in 2011. 

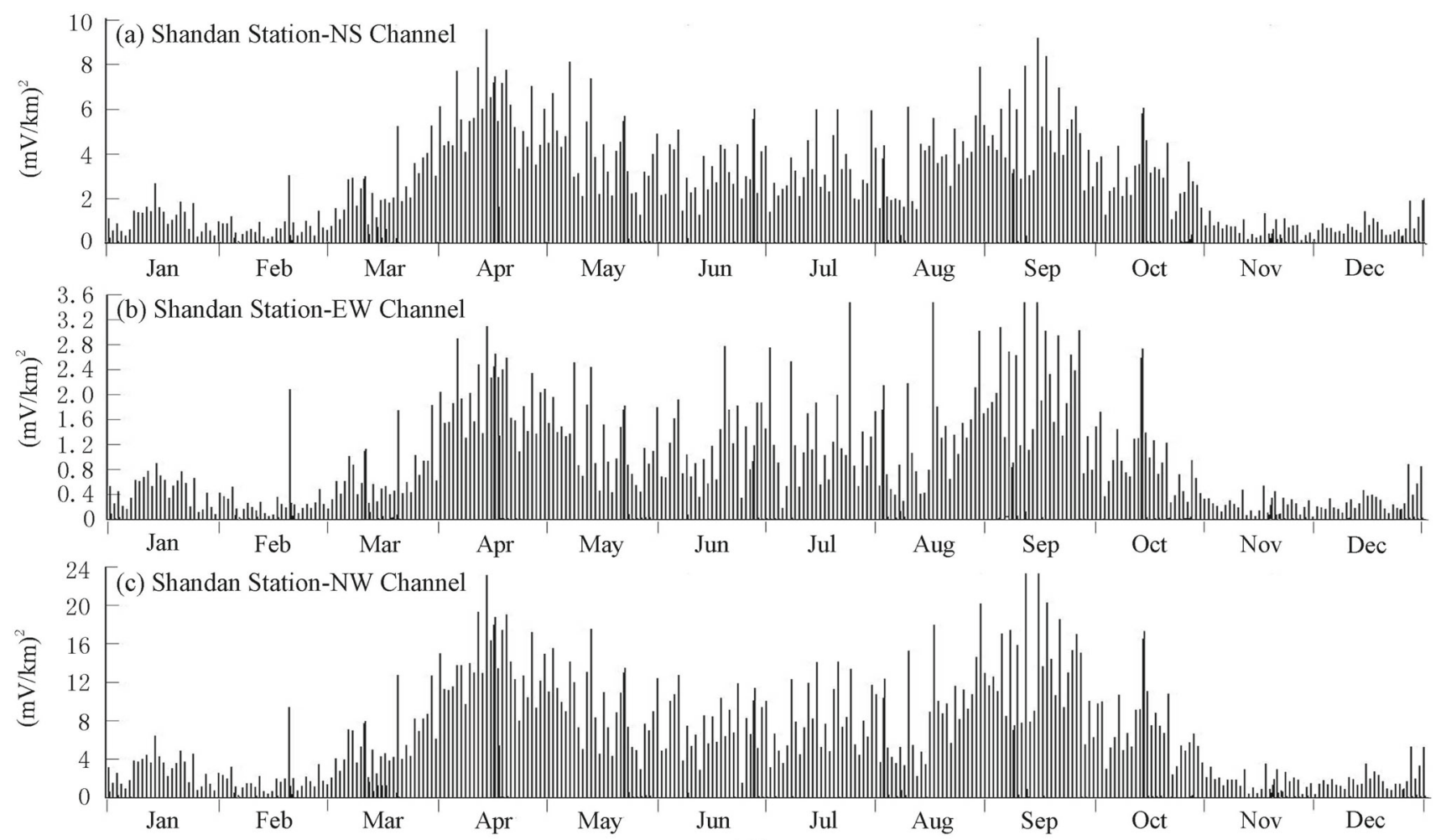

(C)
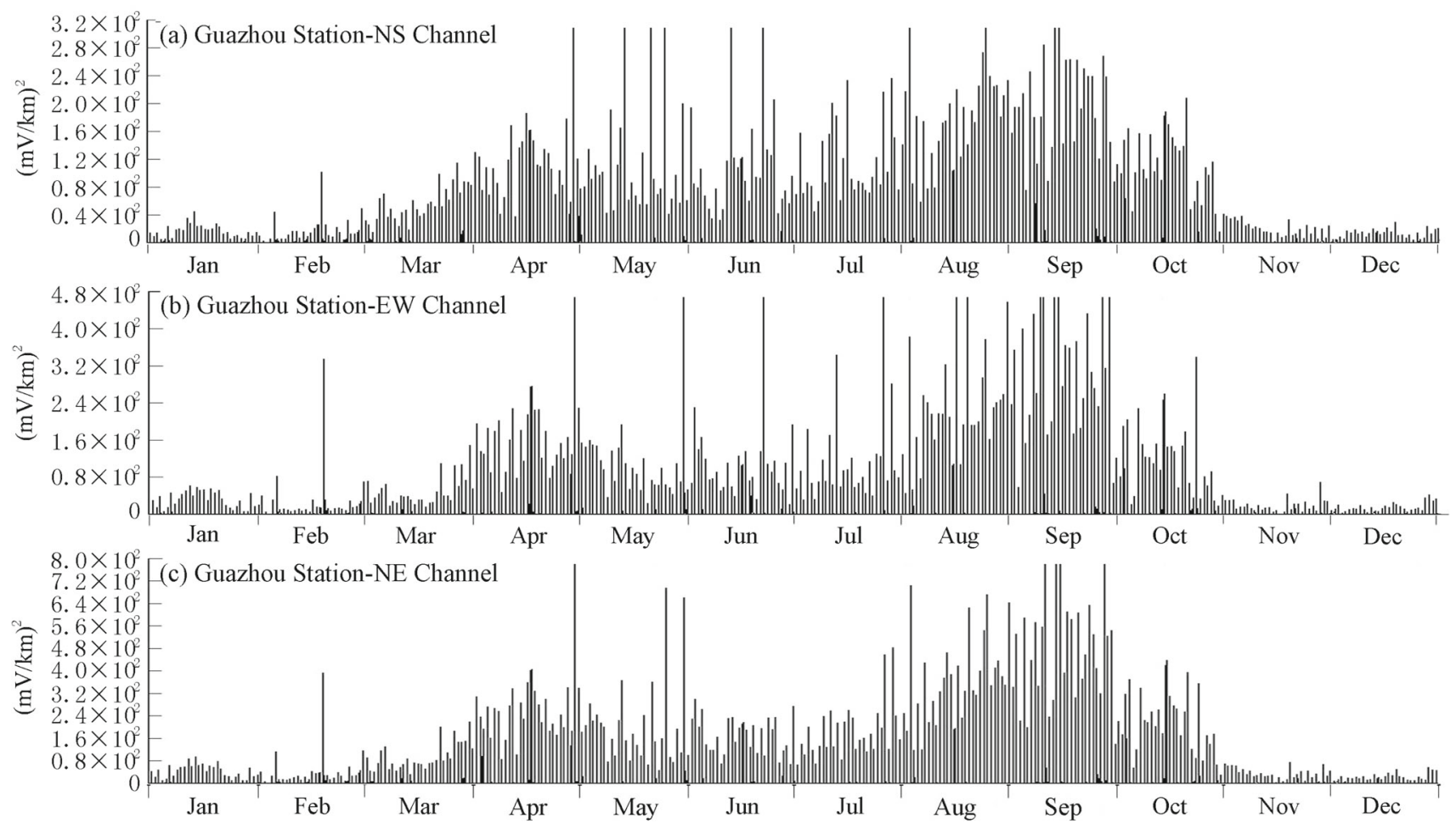

(D)

Figure 4. (Continued.)

location. As shown in the figure, the average value of PSD increased in early March and the increase disappeared in early November. Compared to Pingliang, Gufeng and Shandan geoelectric field stations, we found that the time for the average value of PSD to increase and that to 
decrease was the same, but with slightly different fluctuation. Specifically, the Pingliang, Gufeng and Shandan Stations show faint fluctuation between March-May and August to early November, and the size and shape of the two crests are almost symmetrical based on the axis of early July. In contrast, the fluctuation between August and November was clearly stronger than that between March and May and the PSD also fluctuated greatly, especially at the Guazhou Station.

A comparison among figure $4(\mathrm{~A}-\mathrm{D})$ showed that the four geoelectric field stations had greatly different average value of PSD, and the average value of PSD differed greatly even in the same station but at different directions. An analysis based on previous studies suggested that such differences are associated with the underground electrical structure in the place where the station was located (Fan et al. 2010, 2014; Tan et al. 2012).

Through analysing $K_{\mathrm{p}}$ and $D_{\text {st }}$ indices (figure 3), it is concluded that magnetic indices had no obvious regular fluctuations in 2011, but several magnetic storms occurred all the year round. Upon careful study, the occurrence time of the magnetic storm is not consistent with the PSD fluctuations (figure 4), since the observed data corresponding to the magnetic storm have been deleted during the calculation, so such regular PSD fluctuations are not caused by magnetic storm. No earthquakes over Ms 6.0 had happened within $1000 \mathrm{~km}$ around the above stations in 2011, after carefully investigating the geological condition near the stations. As to Guazhou Station and Pingliang Station that are the farthest from each other are more than 1000 $\mathrm{km}$ away, the same ground interference source will not cause PSD increases that are synchronous from time for two stations that are such far away from each other. The electric field variations were correlated with neither groundwater level variations nor rainfall (Mogi et al. 2000). Thus, it is considered to be an objective natural phenomenon, i.e., seasonal change of the geoelectric field upon preliminary analysis.

Pingliang, Gufeng, Shandan and Guazhou geoelectric field stations located at the northeast edge and east edge of Qinghai-Tibet Plateau and the underground structures of the observation area are basically the same without obvious water system nearby. The maximum entropy spectrum values in 2011 of Changli, Malingshan, Haian and Gaoyou geoelectric field stations that have relatively rich water system located in east Mainland China are computed here to further analyse the influences of underground structure of the observation area on the seasonal changes of geoelectric field (see figure 5 for details).

As shown in the figure, as to Changli Station, the obvious monthly changes have been observed along the EW and NE directions, approximately 25 times a year, and from the NS direction, not only obvious monthly changes have been observed, but also obvious seasonal changes; as to Malingshan Station, obvious seasonal changes have been observed and monthly changes have been observed upon a close-up view as well; as to Gaoyou Station, obvious monthly changes have been observed along the NS direction, but almost no monthly changes along the EW and NE directions, but not obvious seasonal changes can be found upon careful observation; as to Haian Station, obvious monthly changes have been observed along the NS and NE directions, but no obvious monthly changes along the EW direction.

Compared with the four inland stations, it shows that both monthly and seasonal changes can be observed at some of the coastal stations, but only seasonal changes can be observed at inland stations.

\subsection{Geomagnetic field stations}

It is hereby compared with the observation of the geomagnetic field to further study and analyse the monthly and seasonal changes of the geoelectric field. As to computing the PSD values of the geomagnetic field of Jiayuguan, Shandan, Gaoyou and Haian Stations, both computing and drawing methods of them are the same with those of figures 4 and 5, and for specific results see figure 6. Jiayuguan Station and Shandan Station are located in an inland area at the north edge of Qinghai-Tibet Plateau, and Shandan geomagnetic field shares the same observation area with its geoelectric field; Gaoyou and Haian geomagnetic fields are located in the coastal area, sharing the same observation area with Gaoyou and Haiaan geoelectric fields, respectively. As shown in the figure, all components H, D and $\mathrm{F}$ of the four stations including Jiayuguan Station present obvious seasonal changes, and the seasonal changing form of component $\mathrm{H}$ is the most similar to that of geoelectric field, and the other two components are the same with the geoelectric field from the average value of increasing and decreasing time of PSD, but the average value of PSD has no obvious decrease 
from June to July, and no monthly changes have been observed from the above four geomagnetic stations.
The geomagnetic field has variations, tens of gamma in size, with major spectral components at about $24,12,8$ and $6 \mathrm{~h}$ in period, and because of
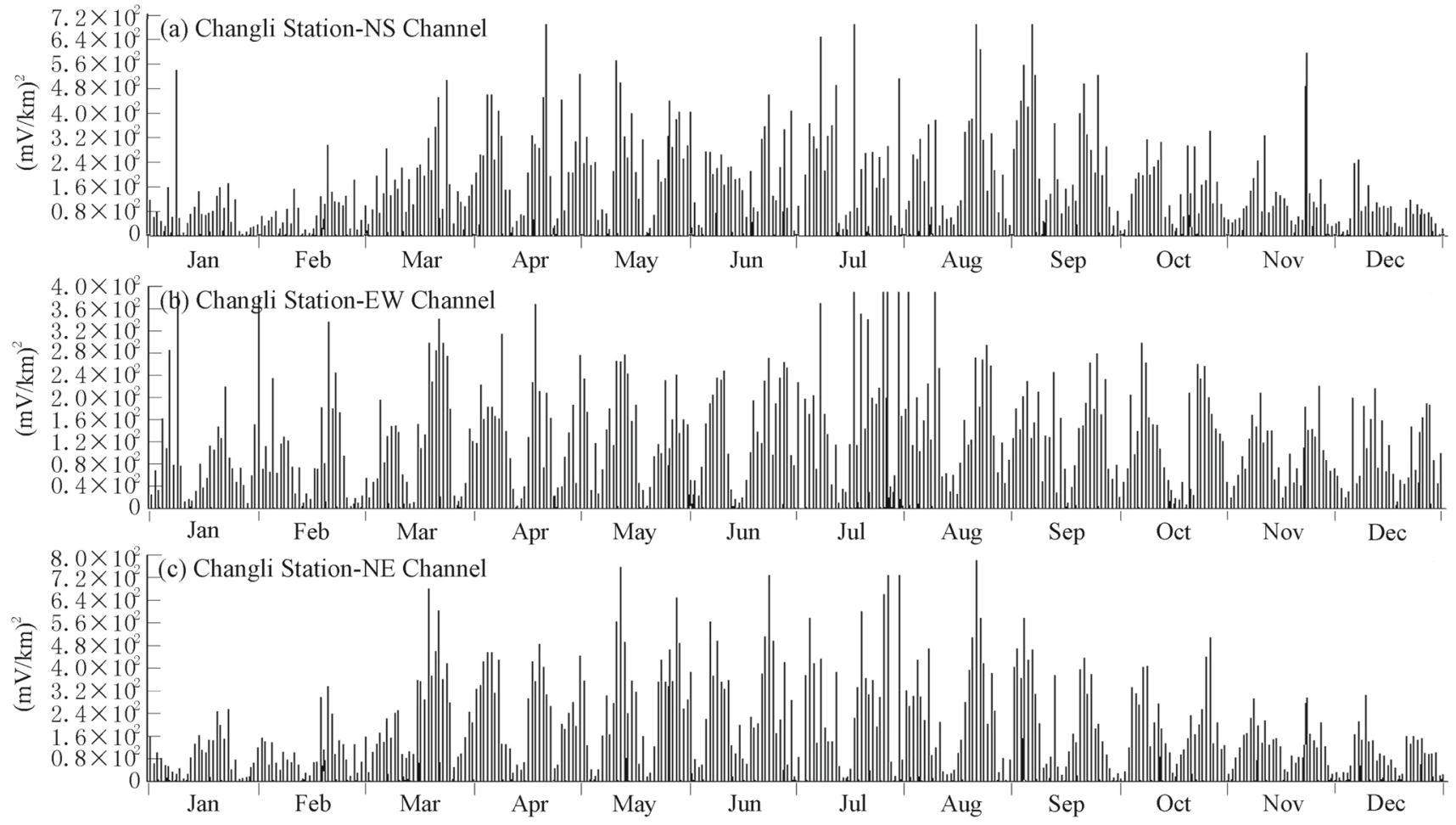

(A)
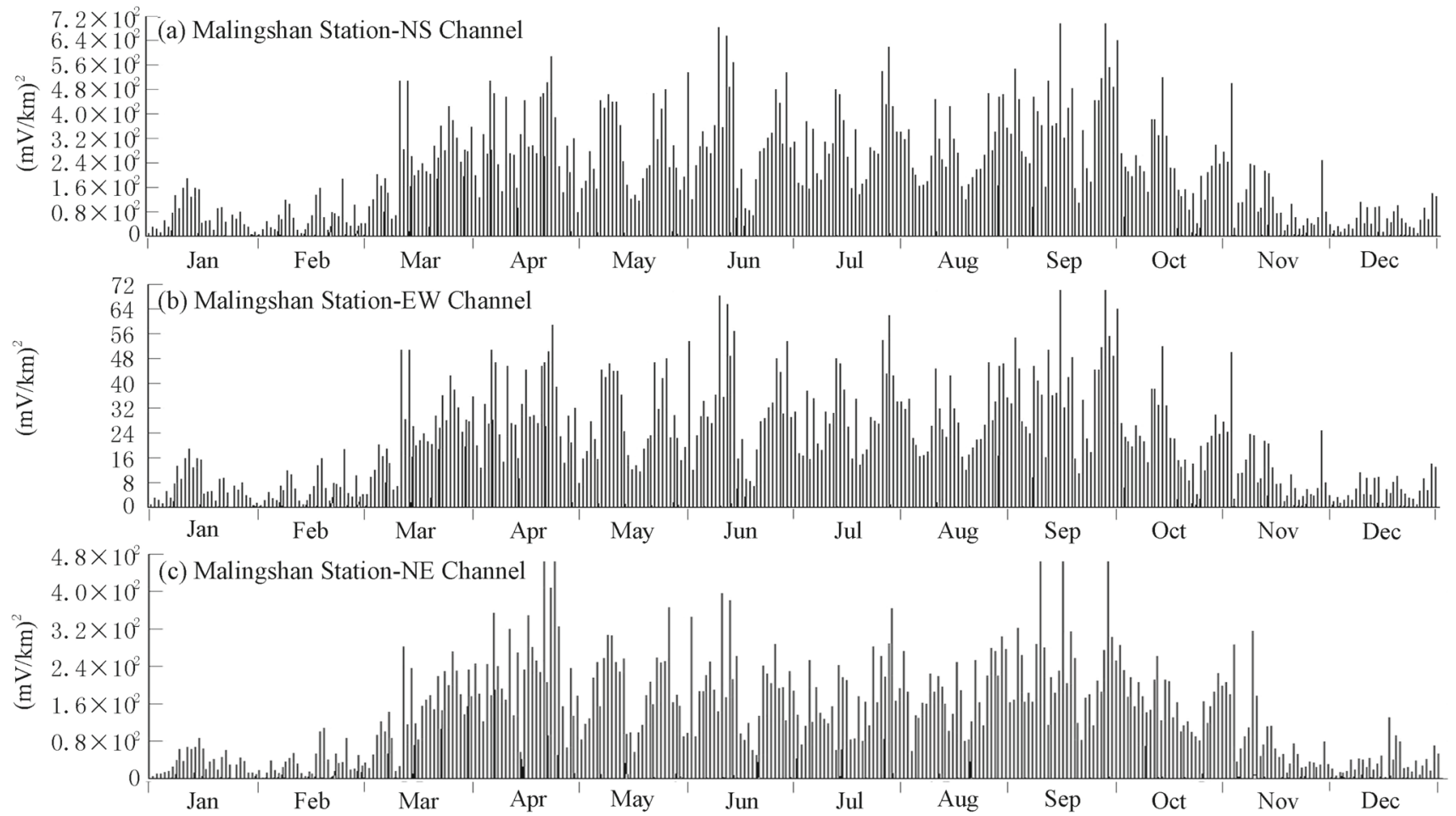

(B)

Figure 5. The map of the averaged PSD for the frequency range 0-0.00832175 Hz of geoelectric fields at the Changli (A), Malingshan (B), Gaoyou (C) and Haian (D) stations in 2011. 

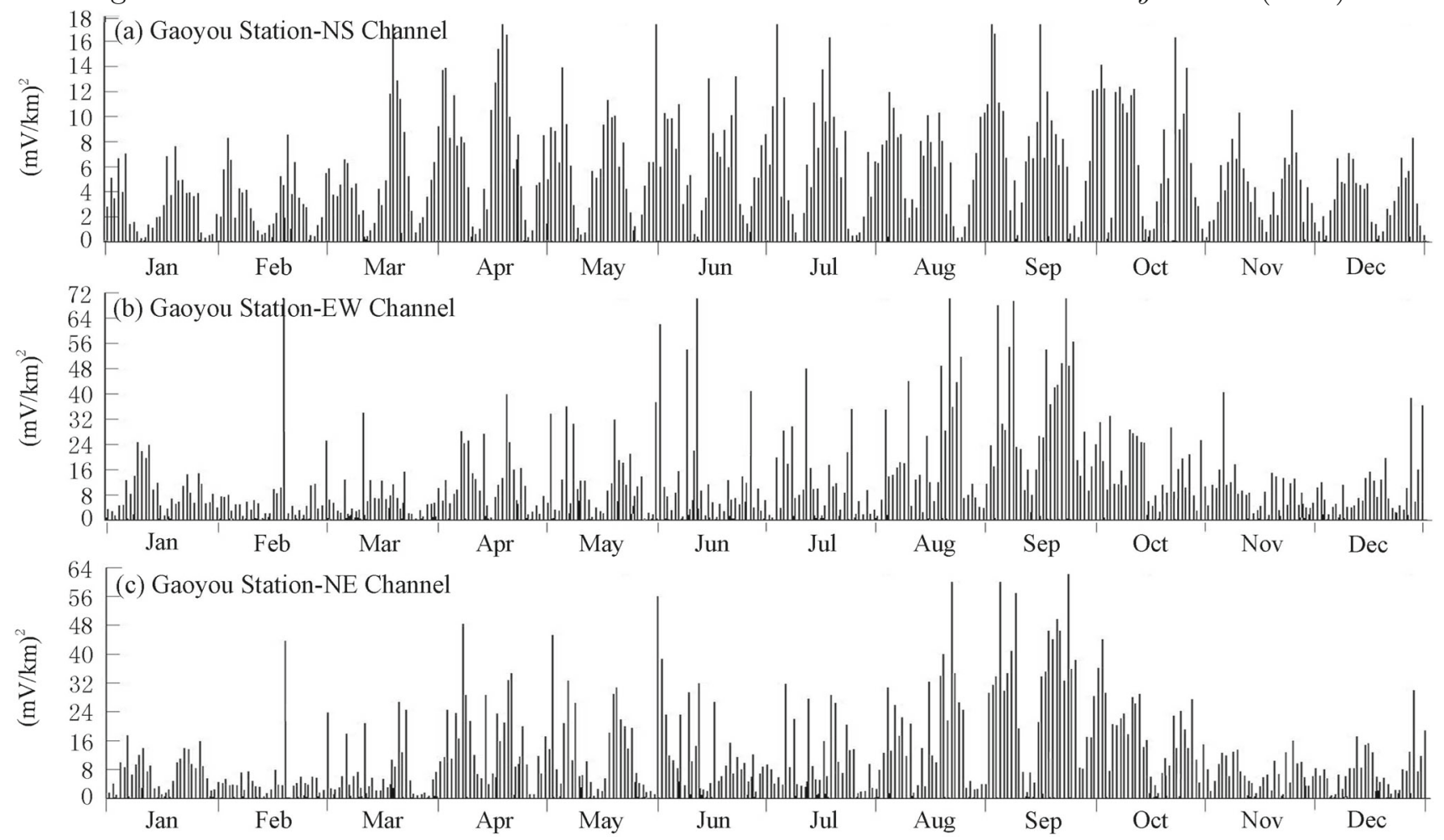

(C)
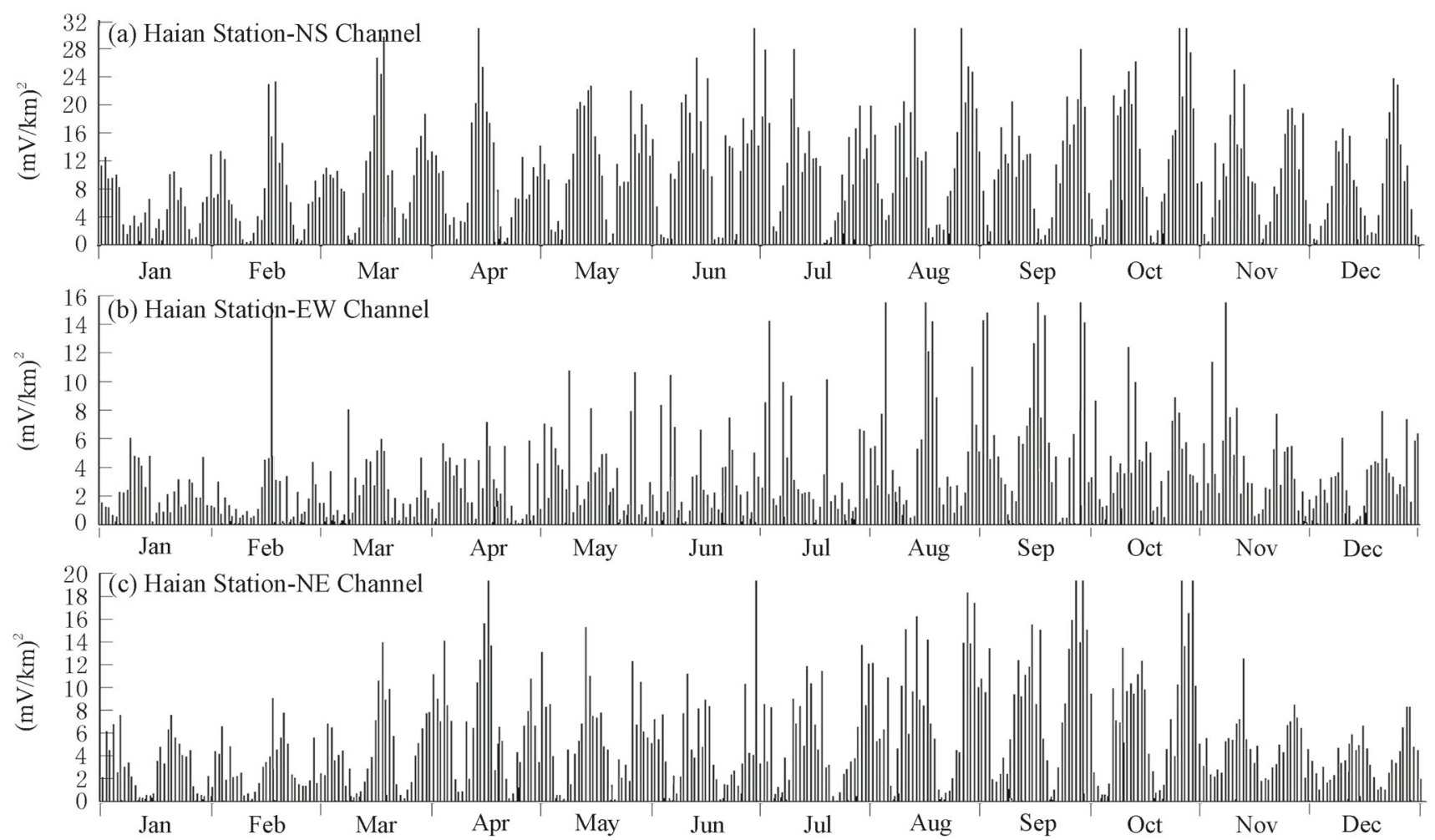

(D)

Figure 5. (Continued.)

the 10.6-yr cycle in solar activity, a similar cycle of geomagnetically quiet years occurs (Campbell 1989). We do not obtain the daily harmonics and longer periods and their amplitude over months/seasons from the figure because of the data length, drawing and so on. 


\section{Discussion}

The geoelectric field consists of the relatively stable natural electric field and the changing electric fields including the telluric electricity field and interference compositions. When the electromagnetic environment and the observation system are relatively perfect, the interference compositions
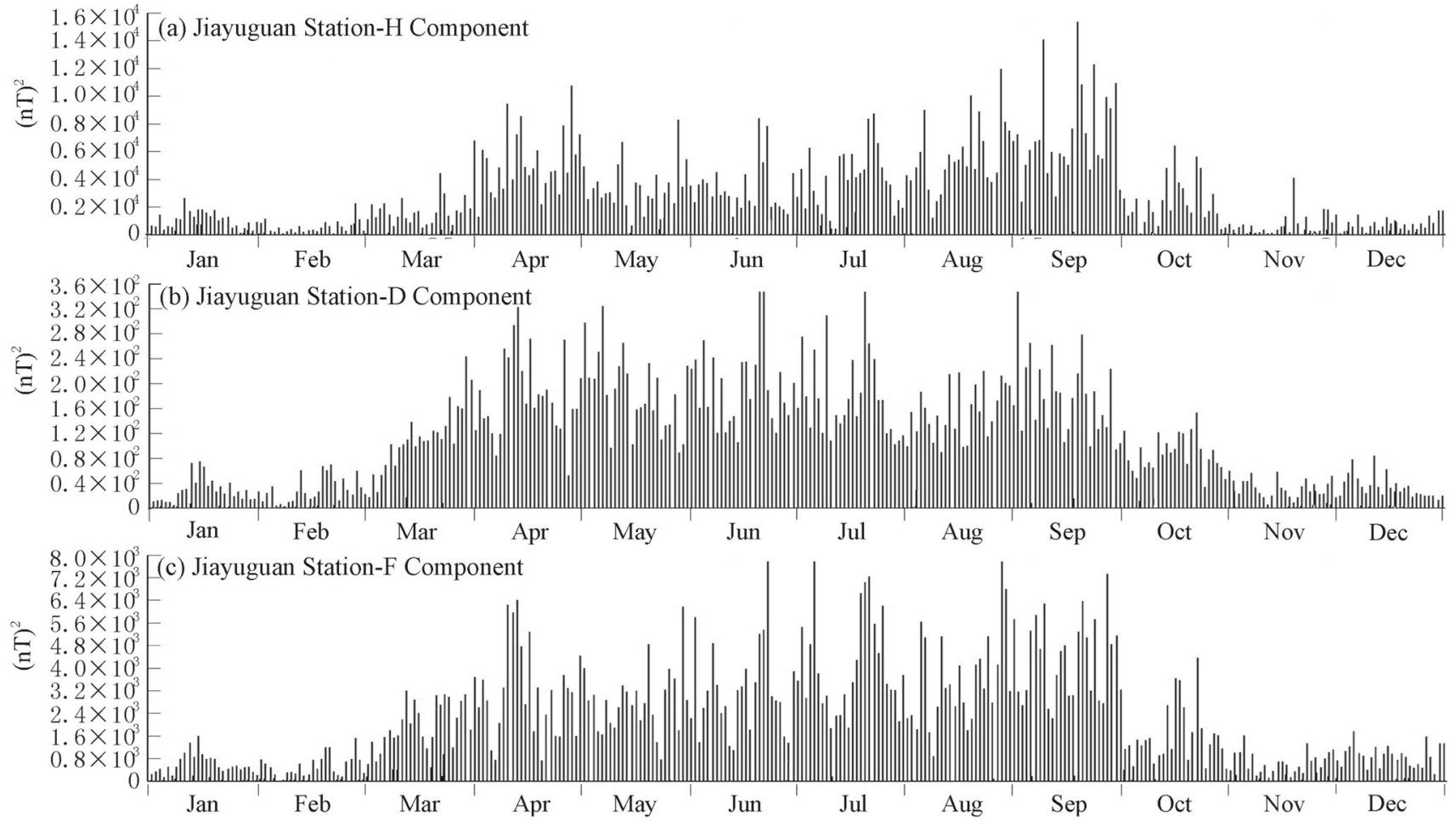

(A)
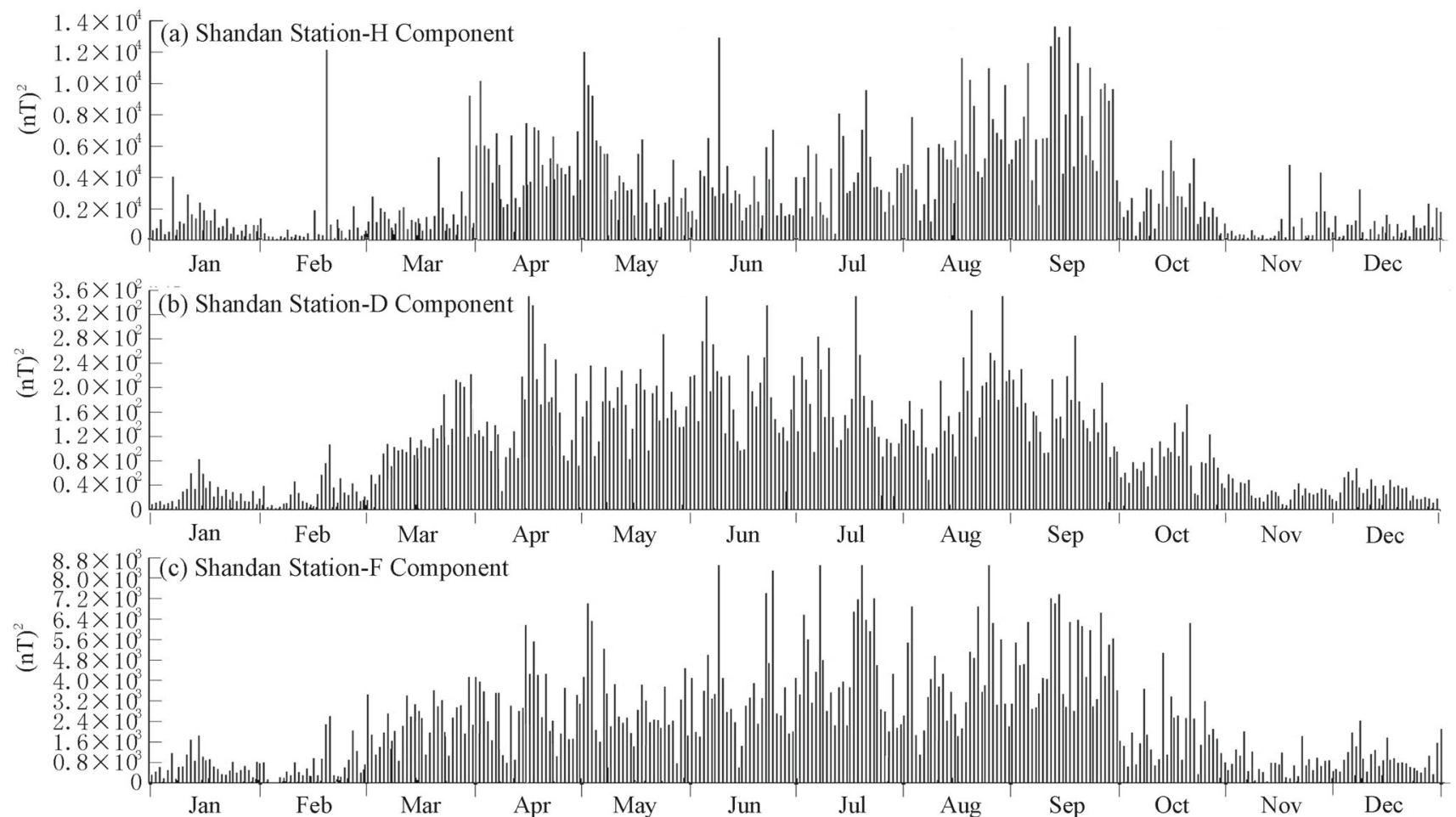

(B)

Figure 6. The map of the averaged PSD for the frequency range 0-0.00832175 Hz of geomagnetic fields at the Jiayuguan (A), Shandan (B), Gaoyou (C) and Haian (D) stations in 2011. 

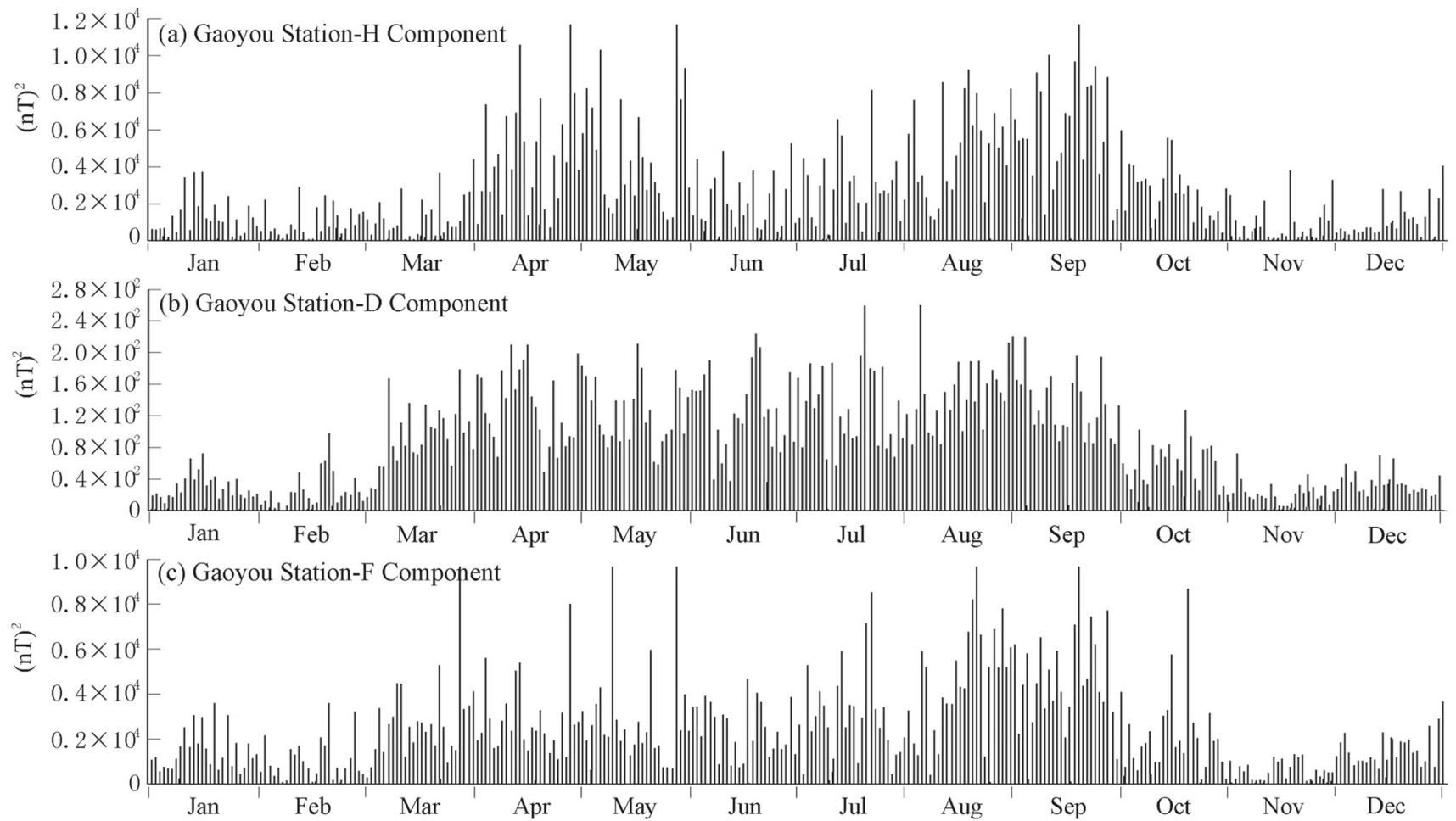

(C)
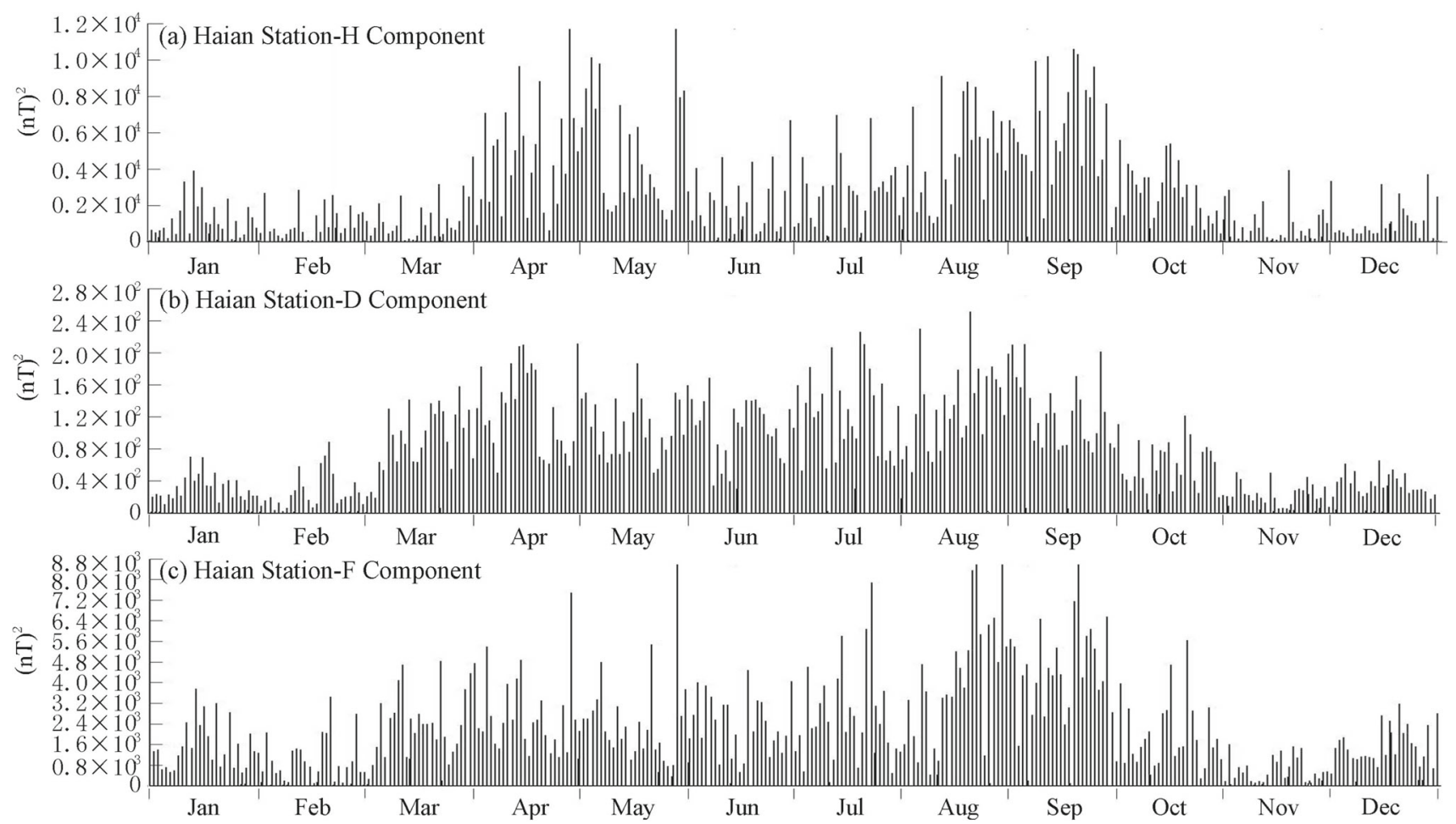

(D)

Figure 6. (Continued.)

may fluctuate in a certain scope. The natural electric field is caused by the physical and chemical actions of the Earth's interior medium, and the telluric electricity field is mainly generated from the space current system and tidal forces.
The conductivity of ionised stratum is associated with the electron density, ion density, the impact frequency of neutral particles and the geomagnetic field. The Earth revolves around the Sun in a nearly circular oval orbit, where the Sun 
is located at the focal point. The Earth passes the perihelion generally in early January every year and the aphelion in early July. As an included angle of $23.5^{\circ}$ exists between the orbital plane and equatorial plane of the Earth, the solar radiation light pressure on the Northern Hemisphere in summer (around July) is stronger than that in winter (around January) which increases the collision frequency of charged particles and the intensity of induced electromagnetic field. In the ground electromagnetic observation, it shows that the beginning of July is the midpoint of high PSD value time period and January is the midpoint of low PSD value time period of the seasonal changes of the electromagnetic field. This point of view also has been proved by Gu (1990) through researching earth rotation and atmospheric angular momentum, and the condition that most of the non-tidal high-frequency changes whose cycle is smaller than 30 days among the daily changes can be explained by the high-frequency excitation caused by the atmosphere to the Earth's rotation.

According to Loyd's seasons in astronomy, March, April, September and October involving vernal equinox and autumnal equinox are expressed as Season E; then May, June, July and August involving summer solstice are expressed as Season J; and November, December, January and February involving winter solstice are expressed as Season D. Concluded from Global Magnetic Network, as to the mid-latitude current vortex intensity of $\mathrm{Sq}$ global equivalent current in the three seasons ( Xu 2000), it is $215 \mathrm{kA}$ for Season D, $240 \mathrm{kA}$ for Season E and $267 \mathrm{kA}$ for Season J. It is obvious that PSD changes of maximum entropy spectrum totally correspond to the changes of overall intensity of current vortex field, and the PSD of geoelectric field of Season $J$ is the maximum, that of Season D is the minimum and that of Season E is changing from weak to strong and from strong to weak.

With the Moon revolving around the Earth and the Earth revolving around the Sun, when the celestial longitude of the Sun is the same as that of the Moon, it is the beginning of the month. A synodic month has 29.5366 days (i.e., 29 days, $12 \mathrm{~h}, 44 \mathrm{~min}$ and $3 \mathrm{~s}$ ), namely, a year has 24.72 cycles, identical with monthly change cycle. The gravitational pull of the Moon on the rotating earth raises the atmosphere and ocean levels both on the side facing the Moon and on the side away from the Moon. This semidiurnal tidal effect occurs because the Moon's attraction overbalances the centrifugal force on the facing side of the Earth, whereas the centrifugal force overbalances the attraction on the away side (Campbell 1989). Tide generating force acts on the oceans and lakes, giving rise to the migration of the water in rock fractures, so as to cause monthly changes of geoelectric field (Tan et al. 2010).

Tan et al. (2012) have studied the daily change waveform of the geoelectric field and found that the daily change waveforms of geoelectric field to be shown in three types: (i) it is shown in smooth sine or near-sine type; (ii) sine form only appears before and after noon; and (iii) it has no daily changes. Daily change waveforms of the geoelectric fields of Pingliang, Gufeng, Shandan and Guazhou Stations are shown in the second type, namely, sine form only appears before and after noon. The geoelectric field stations whose peak-peak value only appears before and after noon often exist in the regions having thick Quaternary sedimentary deposit, high water content in rock and strong water permeability, and it has a close relation with space $\mathrm{Sq}$ current caused by atmospheric tides (Tan et al. 2010), and the generation and change of space current like Sq are mainly influenced by the Sun (Sun and Wang 1990). As to Changli, Malingshan, Haian and Gaoyou geoelectric field stations located in the east Mainland China, daily change waveforms of Changli, Haian (along south-north) and Gaoyou Stations show smooth sine changes, and daily change waveforms of Haian (along east-west), Malingshan as well as Pingliang, Gufeng, Shandan and Guazhou Stations located in inland area show sine changes only happened before and after noon. The trend of the underground crack of the observation area of Haian geoelectric station is nearly south-north, and the peak-peak value of the filtrating electric field along the south-north caused by extruding and drawing of tidal force along the east-west is large, corresponding to change trend of the force along the east-west.

To summarise, waveform changes of the geoelectric field are caused by the combined action of the Moon revolving around the Earth, the Earth revolving around the Sun and electromagnetic induction of ionised stratum, and the reason that geoelectric field shows different waveforms is related with the underground structure of the observation area and its surrounding. Solar and lunar tidal forces will play a predominant role if the observation area and its surrounding have a rich underground water system; otherwise the induction of seasonal changes of various current systems 
of the space outside the Earth in the media of the Earth will play a predominant role.

\section{Conclusions}

In this paper, MEM is used to have analysed and computed observation data of the four geoelectric field stations located at the northeast edge and north edge of Qinghai-Tibet Plateau as well as the four geoelectric field stations located at eastern coastal Mainland China in 2011, and some characteristics of the monthly and seasonal changes of geoelectric fields are concluded upon contrast analysis and study with the observation of geomagnetic field.

(1) The obvious seasonal changes (PSD values increased from the beginning of March, until the early November, such increase disappeared) can be observed by the geoelectric field stations located in inland area; the obvious monthly changes (approximately 25 times a year) can be observed by the geoelectric field stations located in coastal area; and some of them can observe both monthly and seasonal changes.

(2) Components H, D and F of geomagnetic field stations can observe obvious seasonal changes at the same time. Among them, the waveform of the seasonal changes of component $\mathrm{H}$ is most similar to that observed at the geoelectric field. None of the three components have observed monthly changes.

(3) Seasonal changes of geoelectric field are caused by the electromagnetic induction of the seasonal changes of ionospheric current induction intensity inside the Earth generated by the change of relative position between the Earth and the Sun; monthly changes of geoelectric field are caused by migration of water in the cracks under observation area and its surrounding that is caused by the change of relative positions between the Sun, the Earth and the Moon caused by the Moon revolving around the Earth and the Earth revolving around the Sun.

(4) Monthly changes and seasonal changes of the geoelectric field are related to the underground crack and water system of the observation area. When the observation area and its surrounding have a rich water system and the trend of the underground crack is similar to the observed direction, the solar and lunar tidal forces will play a predominant role, namely, it shows monthly changes; otherwise, the induction of seasonal changes of various current systems of the space outside the Earth in the media of the Earth will play a predominant role, namely, it shows seasonal changes.

\section{Acknowledgements}

This work was supported by the Gansu Province Science and Technology Plan Project (17JR5RA338). The authors wish to thank the editor and the reviewer for their fruitful comments on an earlier version of the paper.

\section{References}

An Z H, Du X B, Tan D C, Fan Y Y, Liu J and Cui T F 2013 Study on the geo-electric field variation of Sichuan Lushan Ms 7.0 and Wenchuan Ms 8.0 earthquake; Chin. J. Geophys. 56(11) 3868-3876.

Campbell W H 1989 An introduction to quiet daily geomagnetic fields; Pure Appl. Geophys. 131(3) 315-331.

Danilov A D and Lastoviks J 2001 Effects of geomagnetic storms on the ionosphere and atmosphere; Int. J. Geomagn. Aeron. 2(3) 209-224.

Du X B, Xi J L, Tan D C, Zhao J L, Qian J D, Lu Y Q, Lu J, Tang Y X, Kang H L, Chen Y F and Wang D Z 2006 Specification for the construction of seismic station Geoelectrical station Part 2: Geoelectrical field observatory; The Seismology Trade Standard of the PRC, pp. 2-5 (in Chinese).

Enomo Y, Hashimoto H, Shirai N, Murakami Y, Mogi T and Takada M 2006 Anomalous geoelectric signals possibly related to the $2000 \mathrm{Mt}$. Usu eruption and 2003 TokachiOki earthquake; Phys. Chem. Earth 31 319-324.

Fan Y Y, Du X B, Zlotnicki J, Liu J, An Z H, Chen J Y, Zheng G L and Xie T 2010 The electromagnetic phenomena before the Ms 8.0 Wenchuan Earthquake; Chin. J. Geophys. 53(6) 2887-2898.

Fan Y Y, Xie T, An Z H, Du X B, Liu J and Chen J Y 2014 Electromagnetic phenomena before the Wenchuan Ms 8.0 and Yushu Ms 7.1 earthquakes; Acta Seismol. Sin. 36(2) 235-251.

Fan Y Y, Du X B, Tan D C, An Z H, Liu J and Wang J J 2015 Geoelectrical field variations in Gansu area before the 2013 Lushan Ms 7.0 and Minxian-Zhangxian Ms 6.6 earthquakes; Earthquake 35(1) 100-111 (in Chinese).

Gonzalez W D, Joselyn J A, Kamide Y, Kroehl H W, Rostoker G, Tsurutani B T and Vasyliunas V M 1994 What is a geomagnetic storm? J. Geophys. Res. 99(4) 5771-5792.

Gu Z N 1990 A relation between solar activity and the Earth's rotation; Earth Moon Planets. 48(3) 189-195.

Hattori K, Akinaga Y, Hayakawa M and Uyeda S 2002 ULF magnetic anomaly preceding the 1997 Kagoshima earthquakes; In: Seismo-electromagnetics: Lithosphereatmospherre-ionosphere coupling (eds) Hayakawa M and Molchanov O A, Terr. Sci. Publ., Tokyo, pp. 19-28. 
Huang Q H and Liu T 2006 Earthquake and tide response of geoelectric potential field at the Niijima station; Chin. J. Geophys. 49(6) 1585-1594.

Huang F Q, Li M, Ma Y C, Han Y Y, Tian L, Yan W and Li X F 2017 Studies on earthquake precursors in China: A review for recent 50 years; Geod. Geodyn. 8(1) 1-12.

Israil M, Tyagi D K, Gupta P K and Niwas S 2008 Magnetotelluric investigations for imaging electrical structure of Garhwal Himalayan corridor, Uttarakhand, India; J. Earth Syst. Sci. 117(3) 189-200.

KPAER A 1951 Geoelectrics principle [M]; Geological Publishing House, Beijing, pp. 100-236.

Liu J, Du X B, Zlotnicki J, Fan Y Y, An Z H, Xie T, Zheng G L, Tan D C and Chen J Y 2011 The changes of the ground and ionosphere electric/magnetic fields before several great earthquakes; Chin. J. Geophys. 54(11) 2885-2897.

Ma Q Z, Feng Z S, Song Z P and Zhao W G 2004 Study on the variation characteristics of the geoelectric field preceding earthquakes; Acta Seismol. Sin. 17(3) 334-343.

Miglani R, Shahrukh M, Israil M, Gupta P K, Varshney S K and Elena S 2014 Geoelectric structure estimated from magnetotelluric data from the Uttarakhand Himalaya, India; J. Earth Syst. Sci. 123(8) 1907-1918.

Mogi T, Tanaka Y, Widarto D S, Arsadi E M, Puspito N T, Nagao T, Kanda W and Uyeda S 2000 Geoelectric potential difference monitoring in southern Sumatra, Indonesia - Co-seismic change; Earth Planets Space 52(4) 245-252.

Nagamoto H, Fukushima T, Ida Y, Matsudo $\mathrm{Y}$ and Hayakawa M 2008 Disturbances in VHF/UHF telemetry links as a possible effect of the 2003 Hokkaido Tokachi-oki earthquake; Nat. Hazards Earth Sys. 8(4) 813-817.

Nagao T, Orihara Y, Yamaguchi T, Takahashi I and Hattori K 2000 Co-seismic geoelectric potential changes observed in Japan; Geophys. Res. Lett. 27(10) 1535-1538.

Niwas S, Gupta P K and Gaur V K 2005 Normalized impedance function and the straightforward inversion scheme for magnetotelluric data; J. Earth Syst. Sci. 114(5) 523-531.

Orihara Y, Kamogawa M, Nagao T and Uyeda S 2012 Variations of geoelectric potential differences associated with an anomalous volumetric strain change in the region of expected Tokai Earthquake, Japan; Nat. Hazards Earth Syst. 12(1) 121-127.

Qian J D, Gu Z W and Zhao J L 2004 Technical for the observational environment of seismic stations - Part 2: Electromagnetic observation; The Standard of the PRC, pp. 14-29.

Song Z P, Mei S R, Wu A X and Xue Y 1997 The feature of the maximum entropy spectrum about seismicity before and after disastrous earthquake; Acta Seismol. Sin. 19(1) $7-12$.

Sun Z J and Wang H J 1990 Introduction of geoelectricity; Seismological Press, Beijing, pp. 3-95.
Tan D C, Zhao J L, Xi J L, Du X B and Xu J M 2010 A study on feature and mechanism of the tidal geoelectrical field; Chin. J. Geophys. 53(6) 2887-2898.

Tan D C, Zhao J L, Xi J L, Liu D P and An Z H 2012 The variation of waveform and analysis of composition for the geoelectrical field before moderate or strong earthquakes in Qinghai-Tibetan plateau regions; Chin. J. Geophys. 55(3) 875-885.

Tang J, Zhan Y, Zhao G Z, Deng Q H, Wang J J, Chen X B, Zhao J M and Xuan F 2005 Electrical conductivity structure of the crust and upper mantle along the profile Maqen-Lanzhou-Jingbian in the Northeastern margin of the Qinghai-Tibet Plateau; Chin. J. Geophys. 48(5) 1293-1306.

Uyeda S 1995 Introduction to the VAN method of earthquake prediction. A critical review of VAN; World Scientific Publishing Co., Singapore, pp. 3-28.

Uyeda S, Nagao T, Orihara Y, Yamaguchi T and Takahashi I 2000 Geoelectric potential changes: Possible precursors to earthquakes in Japan; Proc. Nat. Acad. Sci. USA $\mathbf{9 7}(\mathbf{9})$ 4561-4566.

Uyeda S, Hayakawa M, Nagao T, Molchanov O, Hattori K, Orihara Y, Gotoh K, Akinaga Y and Tanaka H 2002 Electric and magnetic phenomena observed before the volcano-seismic activity in 2000 in the Izu Island Region, Japan; Proc. Nat. Acad. Sci. USA 99(11) 7352-7355.

Varotsos P and Alelopoulous K 1984 Physical properties of the variation of the electric field of the earth preceding earthquake I; Tectonophys. 110 73-98.

Wang S Y 1997 The digital signal processing [M]; Beijing Institute Technology Press, Beijing, pp. 274-364.

Xiao Q B, Zhao G Z, Wang J J, Zhan Y and Chen X B 2009 Deep electrical structure of the Sulu orogen and neighboring areas; Sci. China D 52(3) 420-430.

Xu W Y 2000 Geomagnetism; Seismological Press, Beijing, pp. $64-349$.

Zeng Z C, Zhang B, Fang G Y, Wang D F and Yin H J 2009 An analysis of ionospheric variations before the Wenchuan earthquake with DEMETER data; Chin. J. Geophys. 52(1) 13-22.

Zhan Y, Zhao G Z, Unsworth M, Wang L F, Chen X B, Li T, Xiao Q B, Wang J J, Tang J, Cai J T and Wang Y Z 2013 Deep structure beneath the southwestern section of the longmenshan fault zone and seimogenetic context of the 4.20 Lushan Ms 7.0 earthquake; Chin. Sci. Bull. 58(28) 3467-3474.

Zhang X M, Zhai Y Z, Guo X Z and Guo J F 2007 Tidal wave anomalies of geoelectrical field before remote earthquakes; Acta Seismol. Sin. 20(1) 51-62.

Zlotnicki J, Kossobokov V and Mouäl J L 2001 Variation of frequency content of electromagnetic field at the approach and after the 21 July 1995, M=5.7, Yong Deng (China) earthquake; Tectonophys. 334 259-270. 